\title{
Burnout en el servicio de emergencia de un hospital
}

\author{
Georgina Silvia Sarmiento Valverde*1,a
}

\section{RESUMEN}

Con el objetivo de describir la prevalencia del síndrome de burnout en el personal de salud que trabaja en el área de emergencia de un hospital público del Cusco, se realizó un estudio descriptivo transversal analítico en 110 trabajadores, entre médicos, enfermeros, obstetras y personal técnico. Se encontró un $10 \%$ de la población con síndrome de burnout. El grupo de médicos presentó 16,3\% de burnout; el personal de enfermería y obstetras, 8,6 \%; y en el personal técnico de enfermería no se encontró burnout. El personal con contratos temporales, un tiempo de servicio entre 1 a 10 años y de sexo masculino presentaron el síndrome con mayor frecuencia. Se halló un cuadro leve en un $34,5 \%$ y moderado en un $18,2 \%$, asimismo se halló un $15,5 \%$ de nivel alto de agotamiento emocional, un 33,6\% de nivel alto en la dimensión de despersonalización. Finalmente, un 51,8\% de la población presentó una baja realización personal.

Se concluye que uno de cada 10 trabajadores del servicio de emergencia padecía de síndrome de burnout en el momento de la encuesta, que fue mayor en médicos y trabajadores con contratos temporales.

Esta situación, que debe ser corregida, puede influir en la calidad de la atención en estos servicios.

Palabras clave: Síndrome burnout; Personal de salud; Emergencias; Médicos (Fuente DeCS: BIREME).

\section{Burnout in the emergency service of a hospital}

\section{ABSTRACT}

In order to describe the prevalence of the burnout syndrome in the health personnel working in the emergency area of a public hospital in Cusco, a descriptive cross-sectional analytical study was carried out in 110 workers, among which doctors, nurses, obstetricians and technical personnel were included. We found that $10 \%$ of the population suffered from the burnout syndrome. Sixteen point three percent $(16.3 \%)$ of the group of physicians, and $8.6 \%$ of the nurses and obstetricians presented signs and symptoms of burnout, and no burnout was found in nurse technicians. Male personnel with temporary contracts who had a service time between 1 to 10 years suffered more frequently from this syndrome. Among the study population, $34.5 \%$ presented a slight-level burnout syndrome, $18.2 \%$ presented a moderate-level burnout syndrome, $15.5 \%$ had high levels of emotional exhaustion, $33.6 \%$ experienced high levels of depersonalization, and finally $51.8 \%$ presented a low personal fulfillment.

It is concluded that one out of every ten (10) emergency service workers suffered from the burnout syndrome at the time of the survey, which was greater in doctors and workers with temporary contracts.

This situation, which must be corrected, can influence the quality of care in these services.

Keywords: Burnout, professional; Health personnel; Emergencies; Physicians (Source: MeSH NLM).

1. Hospital Nacional Antonio Lorena. Cusco, Perú.

a. Médico Cirujano, Especialista en Medicina Interna.

* Autor corresponsal. 


\section{INTRODUCCIÓN}

El síndrome de burnout se observa con mayor frecuencia en el personal de salud, ya que están en contacto directo con personas enfermas, y experimentan estrés físico, psíquico y emocional (1), es así que este cuadro es visto con mayor frecuencia en el personal de salud de las unidades de cuidados intensivos y de emergencia.

Los médicos, en relación con la población general, tienen más probabilidades de presentar síntomas de burnout (37,9 \% frente a $27,8 \%$ ), y estar insatisfechos con la relación del equilibrio trabajo-vida $(40,2 \%$ frente a $23,2 \%)$ (2). En cuanto al personal de enfermería, de 30 a $40 \%$ sufren burnout frente a un 10 a $15 \%$ de la población general de Estados Unidos ${ }^{(3,4)}$.

A nivel mundial la prevalencia de este síndrome en médicos varía entre $40 \%$ a $60 \%{ }^{(5,6)}$. Se ha observado que los más afectados son los especialistas de medicina interna y emergencias, médicos en los que la prevalencia aumenta con el transcurso de los años y llega, actualmente, a $60 \%{ }^{(7,8)}$. En España, la prevalencia en médicos generales y especialistas se sitúa entre el 30 y el $69 \%{ }^{(9)}$, y en Argentina, llega a $67 \%$, valor que es reportado como alto ${ }^{(10)}$.

En relación al personal de enfermería, a nivel mundial, varía entre un $20 \%$ a $40 \%{ }^{(3)}$. En un estudio mexicano se demostró que la prevalencia se encuentra alrededor del $30 \%{ }^{(11)}$. En nuestro medio el personal de enfermería presenta cuadros moderados y leves ${ }^{(1,12)}$.

A nivel nacional, la mayoría de estudios presenta el síndrome de burnout en grado leve (12-14), salvo un estudio en una clínica particular donde encontraron casos severos en 50,57 \% de la población (15). La prevalencia del burnout se ha reportado en un $12 \%{ }^{(16)}$ y en un $26 \%$ en médicos serumistas en Tacna ${ }^{(17)}$.

Se ha observado que este síndrome es más frecuente en médicos que en el personal de enfermería (18). Los médicos y enfermeras con burnout tendrán altas tasas de abandono de la profesión en un corto tiempo, lo que demuestra que en un futuro habrá menos personal asistencial en los centros hospitalarios (4). Estos trabajadores de emergencia o cuidados críticos están en contacto permanente con pacientes críticamente enfermos lo que provocará que, en algún momento de su vida profesional, tengan repercusiones en la salud física, mental y social, es así que estas áreas de emergencia son clave para la investigación.

Por tal motivo, el objetivo de este estudio fue hallar la prevalencia del burnout en el servicio de emergencia en un hospital público de Cusco, Perú.

\section{EL ESTUDIO}

Diseño y población

Estudio descriptivo transversal analítico que incluyó a todos los trabajadores asistenciales de emergencia que tuvieran una antigüedad mínima de 12 meses de servicio en el Ministerio de salud y una permanencia de 6 meses en el Servicio de Emergencia del hospital Antonio Lorena del Cusco que respondieron a la encuesta del inventario de Maslach Burnout Inventory (MBI) para el síndrome de burnout y una encuesta de factores sociodemográficos. El estudio se realizó entre los meses de octubre a diciembre del año 2017.

\section{DEFINICIONES}

El burnout es un síndrome psicológico que emerge como una respuesta prolongada a los estresores interpersonales crónicos en el trabajo. Las tres dimensiones claves de esta respuesta son agotamiento abrumador, sentimientos de cinismo y desapego del trabajo, con una sensación de ineficacia y falta de realización personal. El significado de este modelo tridimensional es que pone claramente la experiencia del estrés individual dentro de un contexto social e involucra la concepción de sí mismo y de los demás (19).

Servicio de Emergencia: Es la unidad orgánica o funcional en los hospitales que brinda atención médico-quirúrgica de emergencia en forma oportuna y permanente durante las 24 horas del día a todas las personas cuya vida o salud se encuentre en situación de emergencia. Según su nivel de complejidad se podrán resolver diferentes categorías de daños ${ }^{(20)}$.

Inventario de Maslach: El Maslach Burnout Inventory $(\mathrm{MBI})$ es un instrumento de evaluación psicológica de 22 ítems, en los que se plantea al sujeto un conjunto de enunciados sobre sus sentimientos y pensamientos relacionados con diversos aspectos de su interacción continua con el trabajo y su desempeño habitual (21).

\section{ANÁLISIS ESTADÍSTICO}

Para la descripción estadística se calculó la frecuencia absoluta y relativa de las variables nominales y ordinales. El análisis de la frecuencia del síndrome de burnout según variables demográficas se hizo mediante una tabla de contingencia, el cálculo del error estándar y el intervalo de confianza al $95 \%$. Todos los cálculos se hicieron con el programa estadístico SPSS v.24.

\section{RESULTADOS}

Del total de 120 trabajadores de la emergencia, participaron en el estudio 110 (92\%), debido a que algunos se encontraron de vacaciones, otros en capacitación o no pudieron contestar la encuesta. 
En la tabla 1 se muestra la distribución de los grados de

burnout encontrados en esta población. Se observa que, de los 110 trabajadores, $10 \%{ }^{(11)}$ presentó síndrome de burnout severo; $18,2 \%$, moderado; y $34,5 \%$, leve.

Tabla 1. Burnout en trabajadores

\begin{tabular}{|lcc|}
\hline \multicolumn{1}{c}{ Burnout } & Frecuencia & Porcentaje \\
\hline Trabajadores sin burnout & 41 & $37,3 \%$ \\
\hline Burnout leve & 38 & $34,5 \%$ \\
\hline Burnout moderado & 20 & $18,2 \%$ \\
Burnout severo & 11 & $10 \%$ \\
\hline Total & 110 & $100 \%$ \\
\hline
\end{tabular}

En la tabla 2 se observa que 8 médicos $(16,3 \%)$ presentan síndrome de burnout de un total de 49; es así que, entre los médicos el porcentaje de personas con síntomas de burnout es 7,7 puntos porcentuales más alto que el grupo ocupacional de enfermeras y obstetras; finalmente, los técnicos de enfermería no presentaron síntomas del cuadro.

En cuanto a la condición laboral, entre los trabajadores con contratos temporales (Contrato Administrativo de Servicios o CAS, contrato a plazo fijo, locación de servicios y Service), el porcentaje de personas con síntomas de burnout es 15,3 puntos porcentuales más alto que la condición laboral de nombrados.

Con relación al tiempo de servicio, entre los trabajadores que tienen entre 1 a 10 años de servicio el porcentaje de personas con manifestaciones de burnout es 7,8 puntos porcentuales más alto que los trabajadores con un tiempo de servicio de 11 años a más.

Finalmente, en los trabajadores de género masculino el porcentaje de personas con síntomas de burnout es 2,3 puntos porcentuales más alto que en los de género femenino.

Tabla 2. Presencia de síndrome de burnout según características sociodemográficas

\begin{tabular}{|c|c|c|c|}
\hline \multirow[b]{2}{*}{ Características sociodemográficas } & \multicolumn{3}{|c|}{ Síndrome de burnout } \\
\hline & $\mathbf{N}$ & Número de casos & $\%(I C 95 \%)$ de la fila \\
\hline \multicolumn{4}{|l|}{ Grupo ocupacional } \\
\hline Médico & 49 & 8 & $16,3( \pm 10.34)$ \\
\hline Enfermera/Obstetriz & 35 & 3 & $8,6( \pm 9.29)$ \\
\hline Técnico /Auxiliar & 26 & 0 & ,0 \\
\hline \multicolumn{4}{|l|}{ Condición laboral } \\
\hline Nombrado & 76 & 4 & $5,3( \pm 5.04)$ \\
\hline Contratos temporales & 34 & 7 & $20,6( \pm 13.59)$ \\
\hline \multicolumn{4}{|l|}{ Tiempo de servicio en emergencia } \\
\hline De 1 a 10 & 85 & 10 & $11,8( \pm 6.86)$ \\
\hline 11 a más & 25 & 1 & $4,0( \pm 7.68)$ \\
\hline \multicolumn{4}{|l|}{ Sexo } \\
\hline Masculino & 44 & 5 & $11,4( \pm 9.39)$ \\
\hline Femenino & 66 & 6 & $9,1( \pm 6.94)$ \\
\hline
\end{tabular}


Según los resultados encontrados sobre las dimensiones del síndrome de burnout (Figura 1), se halló un 15,5\% de nivel alto en relación al agotamiento emocional; en la dimensión despersonalización se encontró un nivel alto en 33,6 \% de los encuestados; así mismo un 51,8 \% de la población presentó una baja realización personal.

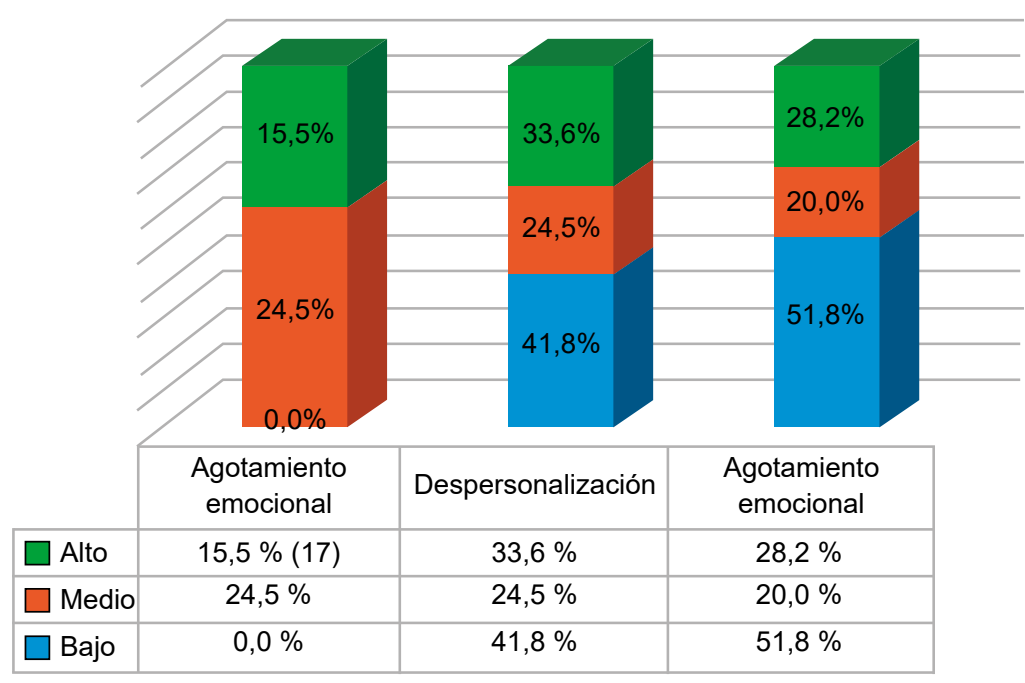

Figura 1. Dimensiones del síndrome de burnout

\section{DISCUSIÓN}

A nivel mundial, los estudios demuestran que el síndrome de burnout cursa con porcentajes elevados, con cifras en promedio del $50 \%$ o mayores ${ }^{(5,22,23)}$. En médicos generales y especialistas la prevalencia llega a ser de $30 \%$ a $69 \%{ }^{(9)}$. En Quito, la prevalencia de burnout es de $39,7 \%$, y es mayor en los servicios de medicina interna y emergencias ${ }^{(18)}$, finalmente, en un estudio realizado en México, el cuadro se presenta en el $52,9 \%$ de los trabajadores del servicio de urgencias (24).

En el Perú, se observó el síndrome de burnout en grado leve en un estudio de 199 (13) $^{\text {y, actualmente, }}$ con el presente estudio se ven que estos porcentajes han aumentado; en esta investigación encontramos 11 trabajadores $(10 \%)$ con criterios de síndrome de burnout, hallazgo importante ya que se dio en todos los profesionales asistenciales de emergencia.

La presente investigación encontró que el $10 \%$ de la población presentó el síndrome de burnout. Este hallazgo es similar a lo reportado en el estudio de Broncano Vargas (1) que demostró un nivel severo del cuadro en $13,3 \%$ de los casos; leve, en $11,1 \%$; y síndrome moderado en 75,6 \% en el personal de enfermería de los servicios de emergencia y cuidados críticos.

En el estudio de Flores Toscana (15), en un grupo de 94 trabajadores asistenciales de emergencia de una clínica particular de Lima, se encontró 50,57 \% de casos de burnout severo, 40,23\% con nivel moderado, y 9,2\% con cuadros leves. Los datos hallados en el presente estudio son mayores que otros observados en hospitales públicos nacionales ${ }^{(12-14)}$, en los que se encontró un nivel de burnout leve en investigaciones que se realizaron en el personal asistencial de los diferentes servicios, lo que demuestra el aumento progresivo del síndrome en los hospitales públicos a nivel nacional, aumento que también se observa en profesionales médicos en todo el mundo ${ }^{(2.6)}$. En nuestra investigación 8 médicos (16,3\%) presentaron síndrome de burnout del total de 49 , seguido por un $8,6 \%$ ( 3 casos) de enfermeras de un total de 30; no se encontraron casos en el grupo de técnicos en enfermería.

El síndrome de burnout fue más frecuente en los médicos que en el personal de enfermería, lo que coincide con otros estudios nacionales e internacionales $(7,13,16)$. Este hallazgo indica que estos profesionales están en riesgo de desarrollar este cuadro, sobre todo, los especialistas en emergencias y cuidados críticos que atienden 
pacientes graves y tienen una mayor carga de trabajo, lo que implica también una mayor responsabilidad.

Asimismo, es importante la relación del síndrome de burnout con la situación laboral del trabajador. Encontramos que se presenta con mayor frecuencia en personas con contratos temporales, con menos tiempo de servicio y en hombres; estos hallazgos se han reportado como significativos en otros estudios.

En el estudio se halló un 20,6 \% de casos en la condición laboral de contrato temporal (contrato administrativo de servicios, plazo fijo y locación de servicios), lo cual influiría en el desarrollo del burnout en el servicio de emergencia, se observa que la situación laboral de ser contratado es un factor de riesgo para el desarrollo del síndrome ${ }^{(13,16)}$. En relación al tiempo de servicio, encontramos $11,8 \%$ de burnout en la población que tiene entre 1 a 10 años de servicio, otro estudio halló que los niveles del síndrome disminuyen con el aumento del tiempo en la profesión ${ }^{(10)}$, es así que un tiempo de servicio mayor o igual de 25 años tendrá menor riesgo de desarrollar burnout ${ }^{(13,16)}$. Estos datos se explicarían por la experiencia y madurez que se adquieren con un mayor tiempo en el trabajo, hacen que el profesional afronte mejor las situaciones estresantes; en contraste, Escribá-Agüira et al. ${ }^{(9)}$ encontraron mayor número de casos en trabajadores con tiempo de servicio mayor a 20 años.

En el presente estudio se encontró un $11,4 \%$ de casos en el género masculino y un $9,1 \%$ en el género femenino, lo que coincide con las investigaciones de Lucero Vasco y Rivera Zambrano ${ }^{(18)}$ y Quiroz ${ }^{(13)}$ en las que el sexo masculino es un factor asociado a presentar el cuadro, en relación al sexo femenino.

En cuanto a las dimensiones del síndrome de burnout, en este estudio se halló un agotamiento emocional alto $(15,5 \%)$, despersonalización alta $(33,6 \%)$, y baja realización personal $(51,8 \%)$. Estos resultados coinciden con Escribá-Agüira et al. ${ }^{(9)}$ en las dimensiones de agotamiento emocional alto (16,5\%), y realización personal baja $(41,1 \%)$; así como también con Lucero y Rivera en la dimensión de agotamiento emocional alto $(27,7 \%)$ y despersonalización alta $(26,7 \%){ }^{(18)}$. Por otro lado, hay una coincidencia parcial con Shanafield et al. $(2,6)$ en las dimensiones despersonalización alta $(29,4 \%)$ y baja realización personal $(34,6 \%)$.

Entre las principales limitaciones de nuestra investigación encontramos la naturaleza descriptiva del estudio, así como el tamaño de la muestra, un tamaño muestral mayor, permitiría hallar o descartar con mayor confianza la presencia de factores asociados.Estos hallazgos de burnout alto en el servicio de emergencia deben ser detectados tempranamente para prevenir y mejorar la calidad de atención.

En conclusión, el síndrome de burnout está en aumento. Los más afectados son el personal médico de sexo masculino con un tiempo de servicio menor o igual a 10 años, y los trabajadores que están en la condición laboral de contratos temporales.

Agradecimientos: A los doctores Alarcón y Palma, y al Servicio de Emergencia del Hospital Antonio Lorena de Cusco.

\section{REFERENCIAS BIBLIOGRÁFICAS}

1. Broncano Vargas YN. Satisfacción laboral y síndrome de Burnout en enfermeras del servicio de emergencia y cuidados críticos del Hospital San Bartolomé 2010. Informe para optar el grado académico de Maestro en Enfermería. Lima: Universidad San Martín de Porres, Facultad de Obstetricia y Enfermería; 2012.

2. Shanafelt TD, Boone S, et al. Burnout and Satisfaction With Work-Life Balance Among US Physicians Relative to the General US Population. Arch Intern Med. 2012; 172(18):1377-85.

3. Aiken LH, Clarke SP, et al. Hospital Care In Five Countries The ways in which nurses' work is structured have left nurses among the least satisfied workers, and the problem is getting worse. Health Affairs. 2001; 20(3):43-53.

4. Aiken L, Clarke S, et al. Hospital Nurse Staffing and Patient Mortality, Nurse Burnout, and Job Dissatisfaction. Health Affairs. 2002; 288(16):1987-93.

5. Tarcan GY, Tarcan M, et al. An analysis of relationship between burnout and job satisfaction among emergency health professionals. Total Quality Management \& Business Excellence. 2016; 28(12):1339-56.

6. Shanafelt TD, Hasan O, et al. Changes in Burnout and Satisfaction With Work-Life Balance in Physicians and the General US Working Population Between 2011 and 2014. Mayo Foundation for Medical Education and Research. 2015; 90(12):1600-13.

7. Golberg R, Wayne Boss R, et al. Burnout and Its correlates in emergency physicians: Four years' experience with a wellness booth. Academic Emergency Medicine. 1996; 3(12): 1157-64.

8. Peckham C. MedScape. [Internet]. 2017 [citado 17 de mayo del 2018]. Disponible en: https://www.medscape.com/features/ slideshow/lifestyle/2017/overview.

9. Escribà-Agüir V, Artazcoz L, Pérez-Hoyos S. Efecto del ambiente psicosocial y de la satisfacción laboral en el síndrome de burnout en médicos especialistas. CIBER Epidemiología y Salud Pública (CIBERESP). 2008; 22(4):300-08.

10. Mariano G. Portales médicos. [Internet]. 2010 [citado 20 de mayo 2018]. Disponible en: https://www.portalesmedicos. com/publicaciones/articles/2544/1/Sindrome-de-burnout-enlos-medicos-.

11. Martinez López C, López Solache G. Características del Síndrome de Burnout en un grupo de enfermeras mexicanas. Archivos en Medicina Familiar. 2005; 7(1): 6-9.

12. Castañeda Camposano SJ, Tineo Gutiérrez CL. Síndrome de 
burnout y su Influencia en la Satisfacción laboral en el Personal de Enfermería del Hospital Carlos Lanfranco la hoz en el distrito de Puente Piedra 2016. Informe para obtar el grado de Licenciado en Psicología. Lima: Universidad Privada Telesup, Facultad de Salud y Nutrición; 2017.

13. Quiroz Valdivia R, Saco Méndez S. Factores asociados al síndrome burnout en médicos y enfermeras del Hospital Nacional Sur Este de ESSALUD del Cusco. SITUA. 2003; 12(23)

14. Salas Aranda P. Frecuencia del Síndrome de burnout y el grado de satisfacción laboral en Internos de Medicina del Hospital Regional Honorio Delgado en el año 2015 - Arequipa. Informe para optener el título profesional de Médico Cirujano. Arequipa; 2016.

15. Flores Toscana MA. Síndrome de burnout en el personal de salud del departamento de emergencia de la Clínica Good Hope Miraflores, 2015. Tesis de Pregrado. Lima: Universidad Privada Arzobispo Loayza, Escuela Profesional de Enfermería; 2016.

16. Valenzuela Salvador AH. Síndrome de burnout de identificación de los factores de riesgo asociados en los trabajadores asistenciales de los establecimientos de salud de la Red de Salud Barranco, Chorrillos, Surco. Tesis de Pregrado. Lima: Universidad Ricardo Palma, Facultad de Medicina Humana; 2010.

17. Valdivia Tapia M. Desempeño laboral y síndrome de burnout en los profesionales médicos Serums de la Red De Servicios de Salud Tacna Minsa 2012. Arequipa; 2013.

18. Lucero Vasco ZM, Rivera Zambrano FX. Relación entre el síndrome de burnout con la satisfacción laboral y el patrón de conducta en médicos residentes y el personal de enfermería del Hospital Eugenio Espejo durante Junio - Julio del 2011. Tesis de Pregrado. Quito: Pontificia Universidad Católica del Ecuador, Facultad de Medicina; 2011.

19. Maslach C, Leiter MP. Understanding the burnout experience: recent research and its implications for psychiatry. World Psychiatry. 2016; 15(2): 103-11.

20. Dirección General de Salud de las Personas. bvs.minsa.gob. pe. [Internet].2006 [citado 20 de mayo 2018]. Disponible en: http://bvs.minsa.gob.pe/local/dgsp/NT042emerg.pdf.
21. Mashlach C, Jackson SE. Maslach Burnout Inventory. 1981.

22. Linzer M, Visser MRM, Oort FJ, Smets EMA, MCMurray JE, M. de Haes HCJ. Predicting and preventing physician burnout: Results from the United States and the Netherlands. Association of Professors of Medicine. 2001:170-75.

23. Busis NA, Shanafelt TD, Keran CM, Levin KH, Schwarz HB, Molano $\mathrm{JR}$, et al. Burnout, career satisfaction, and well-being among US neurologists in 2016. American Academy of Neurology. 2016; 88: 797-08.

24. Guzmán Bautista F. Satisfacción laboral del personal de salud en el servicio de urgencias y su relación con el síndrome de burnout en el Hospital General de zona N. 24 del IMSS. Coyoacán; 2016.

Fuentes de financiamiento:

Este artículo ha sido financiado por la autora.

Conflictos de interés:

La autora declara no tener ningún conflicto de interés.

Correspondencia:

Georgina Sarmiento Valverde

Dirección: Urb. Villa Postal c-15, San Jerónimo - Cusco.

Teléfono: 990028702

Correo electrónico: georgi002@hotmail.com

Recibido: 07 de noviembre de 2018

Evaluado: 04 de diciembre de 2018

Aprobado: 06 de febrero de 2019

(c) La revista. Publicado por Universidad de San Martín de Porres, Perú. (c) в $_{\text {B }}$ Licencia de Creative Commons Artículo en acceso abierto bajo términos de Licencia Creative Commons Atribución 4.0 Internacional. (http://creativecommons.org/licenses/by/4.0/)

\section{ORCID iDs}

Georgina Silvia Sarmiento Valverde (D) https://orcid.org/0000-0002-2053-0557 